\title{
Coronary Artery Calcium Testing in Symptomatic Patients: An Issue of Diagnostic Efficiency
}

\author{
Chad B. McBride • Michael K. Cheezum • \\ Rosco S. Gore • Induruwa N. Pathirana • \\ Ahmad M. Slim • Todd C. Villines
}

Published online: 2 March 2013

(C) Springer Science+Business Media New York (outside the USA) 2013

\begin{abstract}
The detection and quantification of coronary artery calcification (CAC) significantly improves cardiovascular risk prediction in asymptomatic patients. Many have advocated for expanded CAC testing in symptomatic patients based on data demonstrating that the absence of quantifiable CAC in patients with possible angina makes obstructive coronary artery disease (CAD) and subsequent adverse events highly unlikely. However, the widespread use of CAC testing in symptomatic patients may be limited by the high background prevalence of CAC and its low specificity for obstructive CAD, necessitating additional testing ('test layering') in a large percentage of eligible patients. Further, adequately powered prospective studies validating the comparative effectiveness of a 'CAC first' approach with regards to cost, safety, accuracy and clinical outcomes are lacking. Due to marked reductions in patient
\end{abstract}

C. B. McBride • M. K. Cheezum • R. S. Gore • T. C. Villines $(\bowtie)$

Cardiology Service, Walter Reed National Military Medical

Center, 8901 Wisconsin Avenue.,

Bethesda, MD 20850, USA

e-mail: todd.c.villines@health.mil

C. B. McBride

e-mail: chad.b.mcbride@health.mil

M. K. Cheezum

e-mail: Michael.k.cheezum.mil@health.mil

R. S. Gore

e-mail: rosco.gore@med.navy.mil

\section{N. Pathirana}

Department of Medicine, Walter Reed National Military Medical Center, 8901 Wisconsin Avenue.,

Bethesda, MD 20850, USA

e-mail: induruwa.n.pathirana@health.mil

\section{A. M. Slim}

Cardiology Service, San Antonio Military Medical Center,

3551 Roger Brooke Dr.,

San Antonio, TX 78234, USA

e-mail: ahmad.slim@us.army.mil radiation exposure and higher comparative accuracy and prognostic value make coronary computed tomographic angiography the preferred CT-based test for appropriately selected symptomatic patients.

Keywords Coronary artery calcification - Coronary calcium . Cardiac computed tomography $\cdot$ Coronary computed tomography angiography $\cdot$ Chest pain $\cdot$ Stress testing · Guidelines $\cdot$ Coronary artery disease $\cdot$ Coronary heart disease . Cardiovascular risk

\section{Introduction}

Chest pain accounts for approximately 5-6 million emergency department (ED) visits and 1 in every 50 outpatient visits in the United States, ultimately costing more than $\$ 6$ billion dollars in annual healthcare costs [1]. While a majority of presentations for chest pain are ultimately deemed non-cardiac in origin, in light of the substantial morbidity and mortality due to coronary heart disease, most patients undergo non-invasive testing for coronary artery disease (CAD) to better define individual cardiovascular risk. In an era of increasing focus on healthcare resource utilization, considerable interest is aimed at reducing the cost and time to provide appropriate care, ultimately identifying the right test, for the right patient, in the right clinical presentation (assuming a test is needed or indicated at all). Beyond its role as a well-established screening test for subclinical coronary atherosclerosis in asymptomatic patients, coronary artery calcium (CAC) testing has garnered increasing attention as an inexpensive, rapid, reproducible and safe alternative to exclude CAD in symptomatic patients at lowintermediate pre-test risk for obstructive coronary atherosclerosis. Current National Institute for Health and Clinical Excellence (NICE) guidelines recommend testing for CAC 
as the first-line examination in lower risk patients with stable chest pain symptoms $[2 \bullet \bullet$. However, several potential limitations of this approach have curbed the widespread use of CAC testing in symptomatic patients and led to discordant recommendations from other professional societies. These include concerns for the presence of obstructive disease in the absence of CAC, particularly in patients at higher pre-test risk, the high background prevalence of CAC in the general population, and a low-specificity of CAC for obstructive disease, necessitating additional testing in a significant percentage of patients with evident CAC. The concern for diagnostic inefficiency due to test layering in symptomatic patients with positive $\mathrm{CAC}$, as well as the ability to perform coronary computed tomographic angiography (CTA), with proven accuracy and prognostic value at radiation doses comparable to CAC testing in many patients, are prominent limitations to $\mathrm{CAC}$ testing in symptomatic patients in our practice. Herein, we review the evidence supporting CAC testing in symptomatic patients as well as the challenges to its widespread application.

\section{Noninvasive Cardiac Testing - Current Approaches and Limitations}

In order to understand the rationale for utilizing CAC testing in patients with symptoms concerning for angina, one must consider contextually the test performance, cost and current guideline recommendations for standard non-invasive tests for CAD (Fig. 1). Exercise stress electrocardiography (ECG) is commonly performed, relatively inexpensive, can be completed with limited support staff and does not involve ionizing radiation exposure; though it requires an interpretable electrocardiogram and an ambulatory patient. While exercise capacity remains one of the strongest indicators of long-term mortality, the diagnostic accuracy of exercise ECG is low relative to imaging-based tests for CAD. For example, based on pooled summary test performance statistics, exercise ECG has a sensitivity of $68 \%$ and specificity of $77 \%$ for the detection of obstructive CAD on invasive coronary angiography [3]. For this reason, NICE guidelines discourage the use of exercise ECG for the evaluation of patients with stable angina symptoms in patients without known CAD, despite European Society of Cardiology and American College of Cardiology Foundation / American Heart Association (ACCF/AHA) guidelines to the contrary $[2 \bullet \bullet, 4,5 \bullet \cdot$. Indeed, exercise ECG confers the only class I indication for the initial evaluation of suspected stable ischemic heart disease in intermediate risk patients with an interpretable ECG who are able to exercise [5••].

Stress echocardiography and single-photon emission computed tomography (SPECT) both improve diagnostic accuracy for the detection of obstructive coronary artery disease as compared to stress ECG at the expense of time,

\begin{tabular}{|c|c|c|c|c|c|c|c|c|c|}
\hline & \multirow{4}{*}{$\begin{array}{l}\text { Sensitivity } \\
(\%)^{*}\end{array}$} & \multirow{4}{*}{$\begin{array}{l}\text { Specificity } \\
(\%)^{*}\end{array}$} & \multirow{4}{*}{$\begin{array}{c}\text { Mean } \\
\text { Effective } \\
\text { Radiation } \\
\text { Dose } \\
(\mathrm{mSv})\end{array}$} & \multirow{4}{*}{ Cost " } & \multicolumn{5}{|c|}{$\begin{array}{l}\text { Recommendations for Initial Evaluation of } \\
\text { Suspected Ischemic Heart Disease }\end{array}$} \\
\hline & & & & & \multirow{3}{*}{$\begin{array}{c}\text { Possible } \\
\text { ACS }^{`} \\
\text { [16] }\end{array}$} & \multicolumn{4}{|c|}{ Stable Symptoms [5] } \\
\hline & & & & & & \multicolumn{2}{|c|}{$\begin{array}{l}\text { Able to Exercise and } \\
\text { Interpretable ECG }\end{array}$} & \multicolumn{2}{|c|}{$\begin{array}{l}\text { Unable to Exercise or } \\
\text { Uninterpretable ECG }\end{array}$} \\
\hline & & & & & & $\begin{array}{c}\text { Low } \\
\text { Probability }\end{array}$ & $\begin{array}{l}\text { Intermediate } \\
\text { Probability }\end{array}$ & $\begin{array}{c}\text { Low } \\
\text { Probability }\end{array}$ & $\begin{array}{l}\text { Intermediate } \\
\text { Probability }\end{array}$ \\
\hline Stress ECG & 68 & 77 & 0 & $\$ 178.11$ & \multirow{3}{*}{1} & Ila & I & & III \\
\hline $\begin{array}{c}\text { Stress } \\
\text { Echocardiography }\end{array}$ & 85 & 81 & 0 & $\$ 393.18$ & & Ilb & \multirow[t]{2}{*}{ Ila } & Ila & \multirow[t]{2}{*}{ I } \\
\hline SPECT & 88 & 74 & $10-25$ & $\$ 672.28$ & & III & & $\mathbf{u}$ & \\
\hline Coronary CTA & 98 & 89 & $2-10$ & $\$ 262.00$ & IIa & $\mathbf{u}$ & Illb & \multicolumn{2}{|c|}{$\mathrm{Ila} \wedge$} \\
\hline$C A C>0$ & 95 & 50 & $1-3$ & $\$ 46.00$ & $\mathbf{U}$ & \multicolumn{4}{|c|}{$\mathrm{IIb}$} \\
\hline
\end{tabular}

Fig. 1 Comparison of commonly utilized non-invasive tests for coronary artery disease in patients with suspected ischemic heart disease. ACS, acute coronary syndrome; CAC, coronary artery calcium; CTA, computed tomographic angiography; ECG, electrocardiogram; mSv, millisievert; SPECT, single-photon emission computed tomography; U, uncertain. * Per-patient sensitivity and specificity for obstructive coronary artery disease defined as $\geq 50 \%$ coronary luminal narrowing confirmed by invasive coronary angiography according to mean values as reported in current American College of Cardiology Guidelines. Sensitivity for ' $\mathrm{CAC}=0$ ' derived as the weighted mean from Sarwar et al. [24] and Villines et al. [34••]. \# Calendar Year 2012 final Outpatient Prospective Payment System (OPPS)applying procedure codes: Stress ECG (93306), Stress Echo (93351), SPECT (78452), CAC (75571), CCTA (75574), ICA (93458). Note the coverage for coronary artery calcium scanning is limited in many regions. ๆ When follow-up 12-lead ECG and cardiac biomarkers are unremarkable. ${ }^{\wedge}$ In patients able to exercise CCTA meets Class IIa indication if patient (a) has continued symptoms with prior normal test findings (b) has inconclusive results from prior exercise or pharmacological stress testing, or (c) is unable to undergo stress with myocardial perfusion imaging or echocardiography 
resource availability and cost. Of these, stress echocardiography has greater specificity with lower cost and no radiation, but ultimately relies on experienced technicians and readers and adequate image quality to maintain accuracy and reproducibility. While SPECT improves sensitivity for detecting significant $\mathrm{CAD}[6,7]$, it remains more expensive, time consuming and incurs significant radiation, though stress only imaging may limit these drawbacks while providing sufficient negative predictive value (NPV) in lowintermediate risk patients [8].

Lastly, modern coronary CTA has proven to be the most sensitive noninvasive modality to evaluate suspected coronary artery disease in patients with stable or acute chest pain syndromes $[9,10]$. While CCTA exposes patients to ionizing radiation, very low effective doses $(<5$ millisieverts; $\mathrm{mSv}$ ) are now routinely achieved due to advances in scanner technology and image acquisition protocols [11]. Though CCTA requires specialized personnel and equipment that may not be widely available, data has demonstrated the potential for CCTA to reduce costs relative to standard of care primarily in its ability to reduce the time to diagnose patients in the ED setting [12]. Based on its excellent diagnostic accuracy, prognostic value $[13,14 \cdot 15 \cdot]$, and potential to improve the early triage of ED patients, CCTA currently is given a class IIa indication to evaluate lowintermediate risk patients with suspected acute coronary syndrome (ACS) in whom follow-up 12-lead ECG and cardiac biomarkers are normal (Fig. 1) [16].

\section{Coronary Artery Calcium Testing in Symptomatic Patients}

As a non-invasive measure of overall coronary artery disease burden [17], CAC testing is a clinically useful screening test for coronary atherosclerosis. It is currently recommended by ACCF/AHA guidelines in select asymptomatic patients [18•] based on data demonstrating that it significantly improves risk prediction as compared to current global cardiovascular risk scores and the use of highly-sensitive C-reactive protein testing $[19,20]$. Among asymptomatic screening patients, the absence of coronary calcification $(\mathrm{CAC}=0)$ using standard Agatston CAC scoring [21] identifies patients with extremely low rates of subsequent mortality and cardiovascular events [22-24] among patients of diverse age groups [25]. The use of CAC testing in symptomatic patients has traditionally been limited due to fundamental concerns thought to limit its accuracy and/or diagnostic efficiency that include the: (1) occurrence of coronary calcification relatively late in the atherosclerotic process ; (2) high prevalence of CAC in the population and the lack of its specificity for obstructive CAD; (3) demonstration of significant ethnic variability in plaque composition and calcification patterns; and (4) fact that high risk coronary lesions often demonstrate little or no calcification [26-31].

\section{Diagnostic Accuracy of Absent Coronary Calcification}

Studies regarding the diagnostic accuracy of CAC testing in symptomatic patients have generally reported high sensitivity and negative predictive values (NPV) for obstructive CAD in the absence of coronary artery calcification. These values compare favorably to many widely used functional tests for $\mathrm{CAD}$ (Fig. 1) [24]. However, as may be expected, the presence and severity of CAC has limited specificity and positive predictive value for the presence of angiographically significant $C A D$, generally defined as $\geq 50 \%$ lumen stenosis on invasive coronary angiography (ICA). Sarwar and colleagues performed a metaanalysis of 10,355 predominately symptomatic patients undergoing primarily electron-beam computed tomography (EBCT; $\mathrm{n}=8751)$ and demonstrated excellent sensitivity $(98 \%)$ and NPV (93\%) for CAC $>0$ when compared to blinded ICA findings among patients with stable symptoms. Within this cohort, overall specificity of CAC $>0$ was $40 \%$ and positive predictive value (PPV) was $68 \%$ for obstructive CAD. Based on such data, a 2007 ACC/AHA expert consensus statement [32] and recent NICE guidelines [2••] endorsed the use of CAC testing for low-risk, stable symptomatic patients, where CAC testing is used as a filter for further cardiovascular testing in a binary fashion (CAC present or absent). Specifically, patients without $\mathrm{CAC}(\mathrm{CAC}=0)$ avoid further cardiovascular testing $(\mathrm{CAD}$ ruled out) and those with any $\mathrm{CAC}(\mathrm{CAC}>0)$ receive additional testing, such as CCTA, SPECT or ICA, an approach also advocated by some expert opinion [33].

Subsequent studies utilizing multi-detector computed tomography (MDCT) scanners have similarly provided promising data demonstrating generally high, albeit variable, sensitivity and negative predictive values for binary CAC testing among symptomatic patients (Table 1). Recently, investigators from the Coronary CT Angiography Evaluation for Clinical Outcomes: An International Multicenter registry (CONFIRM) evaluated 10,037 symptomatic low-intermediate risk patients undergoing $\geq 64$ slice CCTA and found high sensitivity and NPV for the detection of any stenosis $\geq 50 \%$ (sensitivity $89 \%$, NPV $96 \%$ ) and $\geq 70 \%$ (sensitivity $92 \%$, NPV $99 \%$ ), respectively [34*0]. Within this cohort more than $13 \%$ of patients with $\mathrm{CAC}=0$ had non-obstructive $\mathrm{CAD}$ (purely non-calcified plaque), and $3.5 \%$ and $1.4 \%$ had a stenosis $\geq 50 \%$ and $\geq 70 \%$, respectively. Notably, in both the Sarwar analysis and CONFIRM populations, NPV remained high $(>95 \%)$ despite marked differences in the prevalence of obstructive disease ( $\geq 50 \%$ stenosis), $56 \%$ and $16 \%$, respectively.

However, in reviewing other MDCT studies, the reported sensitivity and NPV of CAC scoring in symptomatic patients are highly variable (Table 1) [35, 36, 37•, 39-41]. Examination of these studies provides a case-study for the effect of pretest probability on test performance in accordance with Bayesian principles. A sub-study of the Coronary Evaluation Using Multi-Detector Spiral Computed Tomography Angiography 
Using 64 Detectors (CORE 64) multi-center trial demonstrated that among 291 high risk symptomatic patients with suspected ACS, $19 \%$ of those with CAC $=0$ had at least one segment of $\geq 50 \%$ stenosis on subsequent ICA [37•]. Similarly, Henneman and colleagues cautioned against the extension of CAC in the ED setting after reporting that among 40 patients presenting with suspected ACS, $12.5 \%(n=5)$ had significant CAD in the absence of calcification [39]. Importantly, these represent smaller studies involving higher-risk patients clinically referred for ICA, a scenario where CAC scoring would generally be inappropriate; and neither study excluded patients with known CAD. In the Henneman paper, for example, $25 \%$ and $28 \%$ of patients had a prior myocardial infarction and percutaneous coronary intervention, respectively. Here, commentary by Blaha et al. regarding the use of Bayes theorem reminds us that as disease prevalence (pretest probability) increases the ability of any non-invasive test to exclude obstructive disease (sensitivity and negative predictive value) is eroded [42].

Given the discrepancy among existing heterogeneous data regarding the accuracy of binary $\mathrm{CAC}$ testing suggests that there is a need for prospective studies assessing the clinical outcomes, cost and safety of this approach prior to widespread clinical adoption of early CAC testing in symptomatic patients. Noting similar sentiment, in the 2012 ACCF/AHA guideline for the diagnosis of patients with potential stable ischemic heart disease, binary CAC testing in symptomatic patients was given a class IIb recommendation (level of evidence ' $\mathrm{C}$ ': may be considered; additional studies are needed; divergence of opinion), with the writing committee stating that "additional evidence in sufficiency large cohorts of patients establishing the uncorrected diagnostic accuracy of CAC to rule in or rule out high-grade coronary artery stenosis in symptomatic patients [is] needed" [5••].

\section{Prognostic Value of a Zero Calcium Score in Symptomatic Patients}

Beyond its proven prognostic value in screening populations and its diagnostic potential in symptomatic patients to rule-out significant $\mathrm{CAD}$ as discussed above, the prognostic value of $\mathrm{CAC}$ testing in symptomatic patients warrants discussion. One of the first studies to evaluate the use of CAC scoring in symptomatic patients in the ED was performed by Georgiou and colleagues [43]. A total of 192 low-intermediate risk patients with suspected CAD underwent calcium scoring in the ED. The treating physician and patient were blinded to $\mathrm{CAC}$ results and subjects were followed up to 7 years. Patients with $\mathrm{CAC}=0$ had an annualized event rate of $0.6 \%$ for a composite of cardiac death, stroke, myocardial infarction, revascularization and hospitalization for angina. The sensitivity and NPV for predicting any cardiac event were both $97 \%$. The Rule Out Myocardial Infarction using Computer Assisted 
Tomography (ROMICAT I) study consisted of 368 symptomatic patients undergoing CCTA and CAC scans for the evaluation of acute chest pain in the ED. Of the 14 patients $(4 \%)$ with purely non-calcified plaque(s), only one (7.1\%) developed ACS, a NPV for binary CAC testing of 99\% [44]. Finally, from among a larger cohort study of more than 1000 patients it was shown that $60 \%$ of low-intermediate risk symptomatic patients referred for stress SPECT had a CAC score of zero, prompting the authors to conclude that these patients could have been discharged without additional evaluation [45]. Cardiac death and ACS occurred in only two $(0.3 \%)$ of the 625 $\mathrm{CAC}=0$ patients over a 7 month follow-up period, providing a sensitivity and NPV of $93.8 \%$ and $99.7 \%$, respectively. On the other hand, $12(1.2 \%)$ of 991 patients with a normal SPECT suffered an event with a sensitivity and NPV of $62.5 \%$ and $98.8 \%$ respectively ( $p=0.04$ for sensitivity). Similar to these findings, a number of other studies attest to the prognostic reassurance provided by the absence of quantifiable CAC on non-contrast CT (Table 2) [34••, 46-53].

It is important to note that when comparing the prognostic value of CAC Agatston scores (plaque burden) versus coronary CTA, which provides angiographic measures of plaque burden, plaque composition and coronary artery stenosis, CAC scoring does not appear to provide significant additional prognostic information among symptomatic patients. Data from the CONFIRM registry [34••] and others [54] have shown that CCTA provides superior prognostic information as compared to CAC testing among symptomatic patients (Fig. 2). Additionally, among patients with obstructive $(\geq 50 \%$ stenosis) disease on CCTA but with $\mathrm{CAC}=0(3.5 \%$ of patients with $\mathrm{CAC}=0)$ within CONFIRM, there was a significant increase in cardiovascular events related to the presence of obstructive CAD (Fig. 3) [34••]. Within this important registry, there was no additional prognostic value for CCTA as compared to CAC scoring in asymptomatic patients [55]. Taken together, these observations appear to support current appropriate use criteria [56] and guidelines for the use of CT-based CAD tests $[4,5 \bullet \bullet, 16$, 18•], highlighting the importance of patient symptoms in this decision-making process.

\section{Limitations of CAC Testing in Symptomatic Patients: A Question of Diagnostic Efficiency}

Based on the data discussed above, when taken in aggregate, CAC testing in symptomatic patients performs reasonably well for excluding significant CAD in symptomatic patients, based primarily on its high sensitivity and NPV. However, widespread endorsement of this approach should be cautioned for several reasons [57]. First, unlike comparable testing options, CAC scoring is a marker of CAD burden and not a direct anatomic or physiologic assessment of stenosis or ischemia, respectively, the primary features that typically guide treatment in symptomatic patients. In addition, based on the fact that calcification occurs relatively late in the atherosclerotic process, CAC testing in younger patients, in whom a smaller percentage of atherosclerosis may be calcified, may lead to false negative tests. For example, it has been reported that up to $47 \%$ of patients at autopsy during their third decade of life have identifiable coronary plaque but only about $3 \%$ of these lesions were calcified [58]. Additionally, symptomatic patients with absent $\mathrm{CAC}$ and significant CAD are more likely to be smokers and have a family history of premature CAD [34••]. Conversely, performance of CAC in older symptomatic patients, a cohort with higher CAC prevalence, may lead to the need for additional testing as a larger percentage of patients will have $\mathrm{CAC}>0$.

\section{Diagnostic Efficiency and Cost Considerations}

Ultimately, the primary issue that should limit the use of CAC testing in symptomatic patients is that of diagnostic inefficiency related to the high rate of additional noninvasive testing (test layering) required for patients with positive CAC when a 'CAC first' approach is applied in low-intermediate risk symptomatic patients. For example, from within the CONFIRM registry, a low-intermediate risk cohort that would generally qualify for CAC testing according to NICE guidelines, $49 \%$ of patients had a CAC score $>0$. Hence, the performance of up-front CAC scoring would have led to approximately half of all patients requiring an early secondary non-invasive test for further evaluation. In practice, we suspect that subsequent testing would generally be stress imaging tests given the limitations of exercise ECG alone, adding to evaluation costs, time to diagnosis and an increase in radiation exposure associated with radionucleotide imaging. In fact, some authors recommend direct invasive coronary angiography for those with high calcium scores $(>400)$ despite atypical symptoms and a lack of any testing suggesting ischemia [33]. An additional consideration that favors coronary CTA as the preferred CTbased test for symptomatic patients, in addition to the improved prognostic performance and lack of significant test layering [59], is the fact that the average radiation exposure for patients undergoing coronary CTA in most experienced centers is $<5 \mathrm{mSv}$ and is often comparable to the effective radiation dose seen in CAC scanning.

Though several peer-reviewed cost comparisons exist for current noninvasive modalities [59-67], similar data comparing $\mathrm{CAC}$ to the usual care of symptomatic patients is limited. While recent NICE guidelines provide an interesting assessment of potential cost savings of a 'CAC first' strategy in low-risk symptomatic patients, it should be cautioned that this cost analysis was primarily aimed toward 


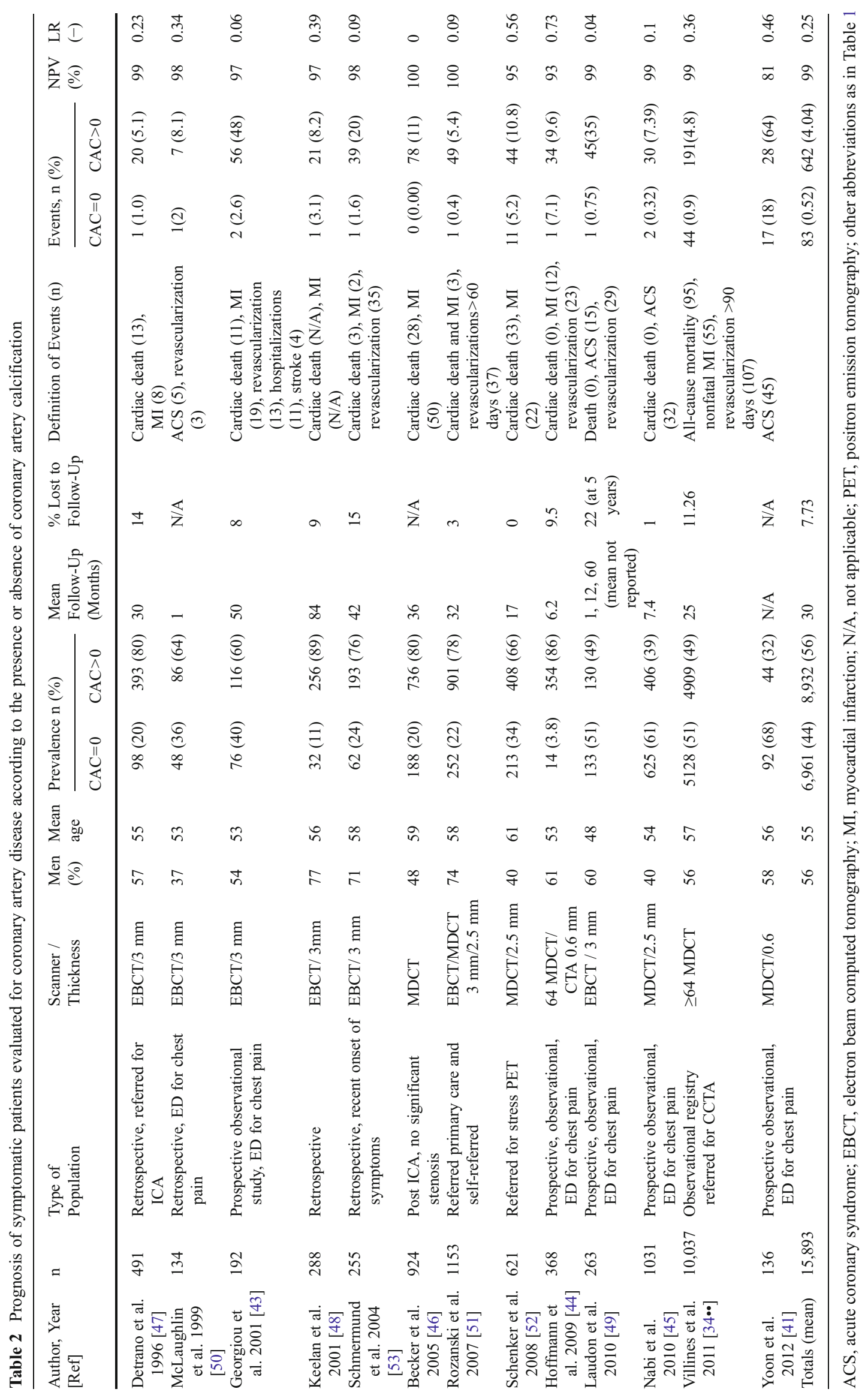






\begin{tabular}{|c|c|c|c|c|}
\hline Model & AUC & SE & $95 \%$ CI & P-value \\
\hline 1 & 0.680 & 0.0173 & $0.646-0.714$ & $<0.001$ \\
\hline 2 & 0.769 & 0.0164 & $0.737-0.801$ & $<0.001$ \\
\hline 3 & 0.825 & 0.0144 & $0.797-0.853$ & $<0.001$ \\
\hline 4 & 0.826 & 0.0146 & $0.797-0.854$ & $<0.001$ \\
\hline
\end{tabular}

Fig. 2 Receiver-operator characteristic curves: major adverse events. Receiver-operator characteristic curves of four models for predicting composite major adverse events in 8907 patients within the CONFIRM registry over a median of 2.1 years of follow-up. Model 2 (Morise score + CAC score) was superior to model 1 (symptoms and risk factors alone by Morise score), $\mathrm{p}<0.001$. Model 3 (Morise score+number of vessels with $\geq 50 \%$ stenosis on $\mathrm{CTA}$ ) was superior to model 2 (risk factors $+\mathrm{CAC}$ score), $\mathrm{p}<0.001$, demonstrating superiority of CTA versus CAC for risk prediction. In addition, when CTA was added to CAC scores, event prediction was improved: Model 4 (Morise score+ CAC score + number of vessels $\geq 50 \%$ stenosis on CTA) superior to Model 2, $\mathrm{p}<0.001$. There was no additional value by adding CAC to models with CTA stenosis: Model 4 not superior to model $3(\mathrm{p}=0.84)$. AUC, area under the receiver-operator curve; CAC, coronary artery calcification; CI, confidence interval; SE, standard error. *Adapted with permission from Villines TC, et al. [34••]
Fig. 3 Major adverse events stratified by presence of coronary artery calcification and stenosis on coronary ct angiography from the confirm registry. CAC, coronary artery calcification; CCTA, coronary computed tomographic angiography; MI, myocardial infarction; Pos, positive for CAC $(\mathrm{CAC}>0)$; Revasc, revascularization occurring $>90$ days following coronary CTA. *Adapted with permission from Villines TC, et al. [34••]

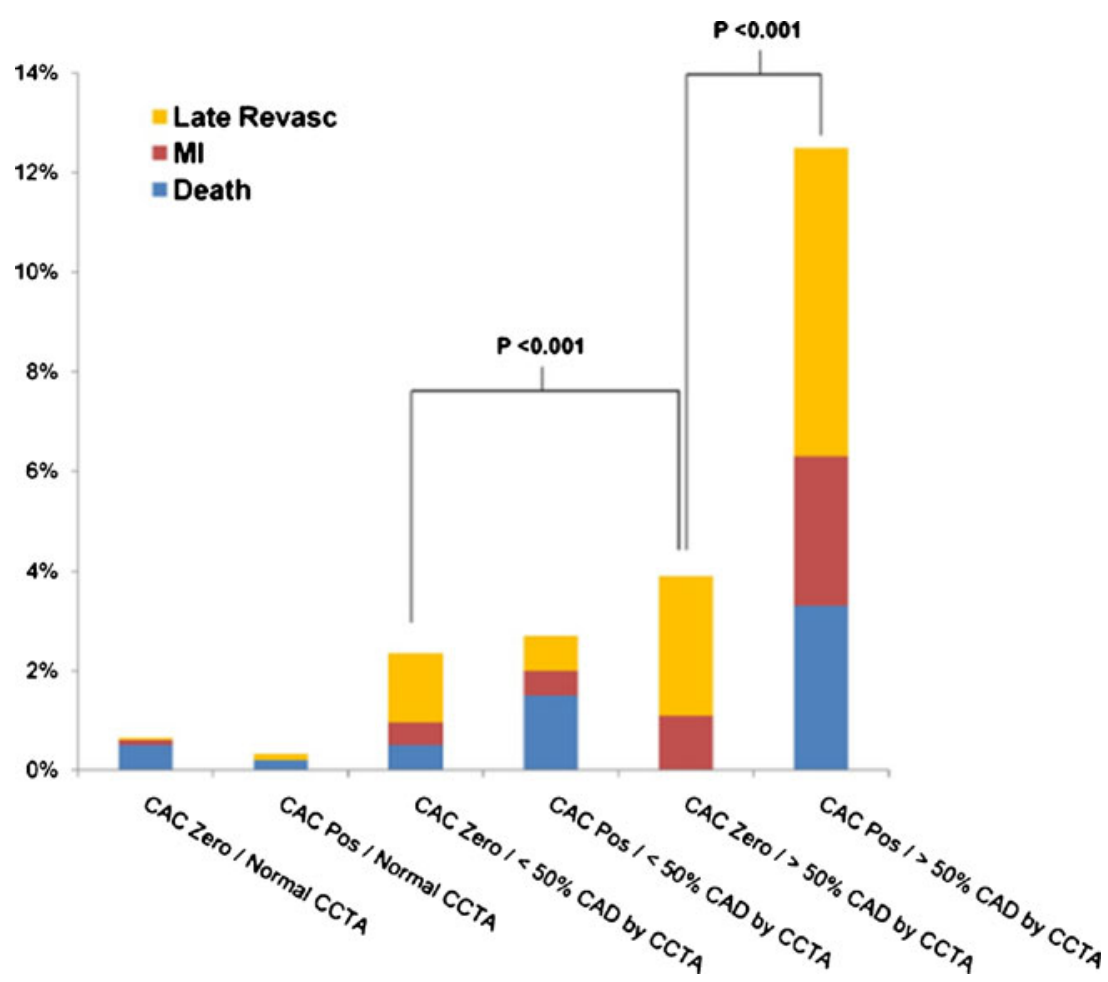


patients with stable chest pain and, thus, should not be extended to patients with acute symptoms, a touted setting for CAC application [2••]. Citing expert opinion, the NICE cost analysis assumed a sensitivity for CTA of $80 \%$ for the detection of stenosis of at least $70 \%$, which is lower than the sensitivites included for both SPECT (86\%) and CAC only (89\%). This assumption ignores significant literature, including several multi-center studies of the accuracy of coronary CTA, reporting coronary CTA as the most sensitive non-invasive test for ruling out significant CAD [10].

Among patients with acute symptoms in whom CAC testing has been potentially advocated for use, we recognize that CAC testing is attractive based on its relatively low cost, reproducibility, ease of performance and interpretation, and potential use after business hours when other modalities requiring more logistical support such as stress echocardiogram, SPECT and coronary CTA may be unavailable. However, it is important to note that the binary use of CAC scanning in symptomatic patients has not been rigorously validated in a large, prospective, multicenter manner. Conversely, coronary CTA has been recently shown in several recent prospective, multi-center trials to be safe, accurate and cost-efficient when performed early for patients with acute chest pain; making it, in our opinion, the preferred testing strategy for patients with acute symptoms not at high pre-test risk who are felt to require further testing [12].

\section{Conclusions}

Coronary artery calcium testing in asymptomatic patients has been shown to significantly improve cardiovascular risk prediction beyond that provided by standard cardiovascular risk variables. Among symptomatic patients, CAC testing has generally high sensitivity and NPV for excluding significant $\mathrm{CAD}$ and subsequent adverse cardiovascular events when performed in low-intermediate risk patients. However, the widespread clinical application of CAC testing in symptomatic patients may be significantly limited by the high prevalence of coronary calcification in the population and low specificity of CAC for obstructive $\mathrm{CAD}$, requiring high rates of additional testing to exclude significant CAD in most symptomatic populations as compared to the use of other standard non-invasive tests. In light of its potential diagnostic inefficiency and the absence of large-scale, prospective studies demonstrating the accuracy, safety and costeffectiveness of a 'CAC-first' approach, and recognizing the progressively lower radiation doses and comparably favorable prognostic information obtained using modern coronary CTA, we feel that coronary CTA is the preferred CTbased test for symptomatic patients.
Acknowledgments Dr. McBride and Dr. Cheezum each contributed equally (co-first authors) to this manuscript.

Conflict of Interest Chad B. McBride declares no conflict of interest. Michael K. Cheezum declares no conflict of interest.

Rosco S. Gore declares no conflict of interest.

Induruwa N. Pathirana declares no conflict of interest.

Ahmad M. Slim declares no conflict of interest.

Todd C. Villines has served on speakers' bureaus for BoehringerIngelheim.

\section{References}

Papers of particular interest, published recently, have been highlighted as:

- Of importance

•- Of major importance

1. Bhuiya FA, Pitts SR, McCaig LF. Emergency department visits for chest pain and abdominal pain: United States, 1999-2008. NCHS Data Brief. 2010; 1-8.

2. • Skinner JS, Smeeth L, Kendall JM, Adams PC, Timmis A. NICE guidance. Chest pain of recent onset: assessment and diagnosis of recent onset chest pain or discomfort of suspected cardiac origin. Heart. 2010;96:974-8. Important explanation of current NICE guidelines for the evaluation of chest pain.

3. Gianrossi R, Detrano R, Mulvihill D, et al. Exercise-induced ST depression in the diagnosis of coronary artery disease. A metaanalysis. Circulation. 1989;80:87-98.

4. Fox K, Garcia MA, Ardissino D, et al. Guidelines on the management of stable angina pectoris: executive summary: The Task Force on the Management of Stable Angina Pectoris of the European Society of Cardiology. Eur Heart J. 2006;27:1341-81.

5. • Fihn SD, Gardin JM, Abrams J, et al. 2012 ACCF/AHA/ACP/ AATS/PCNA/SCAI/STS Guideline for the Diagnosis and Management of Patients With Stable Ischemic Heart Disease: A Report of the American College of Cardiology Foundation/American Heart Association Task Force on Practice Guidelines, and the American College of Physicians, American Association for Thoracic Surgery, Preventive Cardiovascular Nurses Association, Society for Cardiovascular Angiography and Interventions, and Society of Thoracic Surgeons. J Am Coll Cardiol. 2012;60:e44 e164. Updated guidelines on the evaluation and treatment of patients with stable chest pain symptoms and coronary artery disease.

6. Fleischmann KE, Hunink MG, Kuntz KM, Douglas PS. Exercise echocardiography or exercise SPECT imaging? A meta-analysis of diagnostic test performance. JAMA. 1998;280:913-20.

7. Heijenbrok-Kal MH, Fleischmann KE, Hunink MG. Stress echocardiography, stress single-photon-emission computed tomography and electron beam computed tomography for the assessment of coronary artery disease: a meta-analysis of diagnostic performance. Am Heart J. 2007;154:415-23.

8. Duvall WL, Wijetunga MN, Klein TM, et al. Stress-only Tc-99m myocardial perfusion imaging in an emergency department chest pain unit. J Emerg Med. 2012;42:642-50.

9. Samad Z, Hakeem A, Mahmood SS, et al. A meta-analysis and systematic review of computed tomography angiography as a diagnostic triage tool for patients with chest pain presenting to the emergency department. J Nucl Cardiol. 2012;19:364-76.

10. Schuetz GM, Zacharopoulou NM, Schlattmann P, Dewey M. Metaanalysis: noninvasive coronary angiography using computed tomography versus magnetic resonance imaging. Ann Intern Med. 2010;152:167-77. 
11. Vorobiof G, Achenbach S, Narula J. Minimizing radiation dose for coronary CT angiography. Cardiol Clin. 2012;30:9-17.

12. D'Ascenzo F, Cerrato E, Biondi-Zoccai G, et al. Coronary computed tomographic angiography for detection of coronary artery disease in patients presenting to the emergency department with chest pain: a meta-analysis of randomized clinical trials. Imaging: Eur Heart J Cardiovasc; 2012.

13. Cheezum MK, Hulten EA, Fischer C, Smith RM, Slim AM, Villines TC. Prognostic value of coronary CT angiography. Cardiol Clin. 2012;30:77-91.

14. - Hulten EA, Carbonaro S, Petrillo SP, Mitchell JD, Villines TC. Prognostic value of cardiac computed tomography angiography: a systematic review and meta-analysis. J Am Coll Cardiol. 2011;57:1237-47. First large-scale meta-analysis demonstrating the prognostic value of coronary CT angiography.

15. • Min JK, Dunning A, Lin FY, et al. Age- and sex-related differences in all-cause mortality risk based on coronary computed tomography angiography findings results from the International Multicenter CONFIRM (Coronary CT Angiography Evaluation for Clinical Outcomes: An International Multicenter Registry) of 23,854 patients without known coronary artery disease. J Am Coll Cardiol. 2011;58:849-60. Additional data demonstrating the prognostic power of coronary CTA from a large, multinational prospective registry.

16. Wright RS, Anderson JL, Adams CD, et al. 2011 ACCF/AHA focused update incorporated into the ACC/AHA 2007 Guidelines for the Management of Patients with Unstable Angina/Non-STElevation Myocardial Infarction: a report of the American College of Cardiology Foundation/American Heart Association Task Force on Practice Guidelines developed in collaboration with the American Academy of Family Physicians, Society for Cardiovascular Angiography and Interventions, and the Society of Thoracic Surgeons. J Am Coll Cardiol. 2011;57:e215-367.

17. Sangiorgi G, Rumberger JA, Severson A, et al. Arterial calcification and not lumen stenosis is highly correlated with atherosclerotic plaque burden in humans: a histologic study of 723 coronary artery segments using nondecalcifying methodology. J Am Coll Cardiol. 1998;31:126-33.

18. - Greenland P, Alpert JS, Beller GA, et al. 2010 ACCF/AHA guideline for assessment of cardiovascular risk in asymptomatic adults: a report of the American College of Cardiology Foundation/American Heart Association Task Force on Practice Guidelines. J Am Coll Cardiol. 2010;56:e50-e103. An important recent guideline regarding the role of coronary artery calcium scoring as well as other tests and methods for risk assessment in asymptomatic patients.

19. Blaha MJ, Budoff MJ, DeFilippis AP, et al. Associations between C-reactive protein, coronary artery calcium, and cardiovascular events: implications for the JUPITER population from MESA, a population-based cohort study. Lancet. 2011;378:684-92.

20. Budoff MJ, Shaw LJ, Liu ST, et al. Long-term prognosis associated with coronary calcification: observations from a registry of 25,253 patients. J Am Coll Cardiol. 2007;49:1860-70.

21. Agatston AS, Janowitz WR, Hildner FJ, Zusmer NR, Viamonte Jr M, Detrano R. Quantification of coronary artery calcium using ultrafast computed tomography. J Am Coll Cardiol. 1990; $15: 827-32$.

22. Blaha M, Budoff MJ, Shaw LJ, et al. Absence of coronary artery calcification and all-cause mortality. JACC Cardiovasc Imaging. 2009;2:692-700.

23. Budoff MJ, McClelland RL, Nasir K, et al. Cardiovascular events with absent or minimal coronary calcification: the Multi-Ethnic Study of Atherosclerosis (MESA). Am Heart J. 2009; 158:554-61.

24. Sarwar A, Shaw LJ, Shapiro MD, et al. Diagnostic and prognostic value of absence of coronary artery calcification. JACC Cardiovasc Imaging. 2009;2:675-88.
25. Tota-Maharaj R, Blaha MJ, McEvoy JW, et al. Coronary artery calcium for the prediction of mortality in young adults $<45$ years old and elderly adults $>75$ years old. Eur Heart J. 2012;33:2955-62.

26. Bild DE, Detrano R, Peterson D, et al. Ethnic differences in coronary calcification: the Multi-Ethnic Study of Atherosclerosis (MESA). Circulation. 2005;111:1313-20.

27. Detrano R, Guerci AD, Carr JJ, et al. Coronary calcium as a predictor of coronary events in four racial or ethnic groups. N Engl J Med. 2008;358:1336-45.

28. Lee TC, O'Malley PG, Feuerstein I, Taylor AJ. The prevalence and severity of coronary artery calcification on coronary artery computed tomography in black and white subjects. J Am Coll Cardiol. 2003;41:39-44.

29. Marwan M, Ropers D, Pflederer T, Daniel WG, Achenbach S. Clinical characteristics of patients with obstructive coronary lesions in the absence of coronary calcification: an evaluation by coronary CT angiography. Heart. 2009;95:1056-60.

30. Motoyama S, Sarai M, Harigaya $\mathrm{H}$, et al. Computed tomographic angiography characteristics of atherosclerotic plaques subsequently resulting in acute coronary syndrome. J Am Coll Cardiol. 2009;54:49-57.

31. Pflederer T, Marwan M, Schepis T, et al. Characterization of culprit lesions in acute coronary syndromes using coronary dualsource CT angiography. Atherosclerosis. 2010;211:437-44.

32. Greenland P, Bonow RO, Brundage BH, et al. ACCF/AHA 2007 clinical expert consensus document on coronary artery calcium scoring by computed tomography in global cardiovascular risk assessment and in evaluation of patients with chest pain: a report of the American College of Cardiology Foundation Clinical Expert Consensus Task Force (ACCF/AHA Writing Committee to Update the 2000 Expert Consensus Document on Electron Beam Computed Tomography) developed in collaboration with the Society of Atherosclerosis Imaging and Prevention and the Society of Cardiovascular Computed Tomography. J Am Coll Cardiol. 2007;49:378-402.

33. Nasir K, Clouse M. Role of nonenhanced multidetector CT coronary artery calcium testing in asymptomatic and symptomatic individuals. Radiology. 2012;264:637-49.

34. • Villines TC, Hulten EA, Shaw LJ, et al. Prevalence and severity of coronary artery disease and adverse events among symptomatic patients with coronary artery calcification scores of zero undergoing coronary computed tomography angiography: results from the CONFIRM (Coronary CT Angiography Evaluation for Clinical Outcomes: An International Multicenter) registry. J Am Coll Cardiol. 2011;58:2533-40. Report from the CONFIRM coronary CTA registry assessing the prevalence of non-calcified plaque and coronary artery stenosis in symptomatic patients without corononary artery calcification.

35. Akram K, O'Donnell RE, King S, Superko HR, Agatston A, Voros $\mathrm{S}$. Influence of symptomatic status on the prevalence of obstructive coronary artery disease in patients with zero calcium score. Atherosclerosis. 2009;203:533-7.

36. Fernandez-Friera L, Garcia-Alvarez A, Bagheriannejad-Esfahani F, et al. Diagnostic value of coronary artery calcium scoring in lowintermediate risk patients evaluated in the emergency department for acute coronary syndrome. Am J Cardiol. 2011;107:17-23.

37. - Gottlieb I, Miller JM, Arbab-Zadeh A, et al. The absence of coronary calcification does not exclude obstructive coronary artery disease or the need for revascularization in patients referred for conventional coronary angiography. J Am Coll Cardiol. 2010;55:627-34. Study of high-risk symptomatic patients demonstrating a high rate of obstructive CAD in patients without coronary artery calcification.

38. Haberl R, Tittus J, Bohme E, et al. Multislice spiral computed tomographic angiography of coronary arteries in patients with suspected coronary artery disease: an effective filter before catheter angiography? Am Heart J. 2005;149:1112-9. 
39. Henneman MM, Schuijf JD, Pundziute G, et al. Noninvasive evaluation with multislice computed tomography in suspected acute coronary syndrome: plaque morphology on multislice computed tomography versus coronary calcium score. J Am Coll Cardiol. 2008;52:216-22.

40. von Ziegler F, Brendel M, Uebleis C, et al. SPECT myocardial perfusion imaging as an adjunct to coronary calcium score for the detection of hemodynamically significant coronary artery stenosis. BMC Cardiovasc Disord. 2012;12:116.

41. Yoon YE, Chang SA, Choi SI, et al. The absence of coronary artery calcification does not rule out the presence of significant coronary artery disease in Asian patients with acute chest pain. Int J Cardiovasc Imaging. 2012;28:389-98.

42. Blaha MJ, Blumenthal RS, Budoff MJ, Nasir K. Understanding the utility of zero coronary calcium as a prognostic test: a Bayesian approach. Circ Cardiovasc Qual Outcome. 2011;4:253-6.

43. Georgiou D, Budoff MJ, Kaufer E, Kennedy JM, Lu B, Brundage BH. Screening patients with chest pain in the emergency department using electron beam tomography: a follow-up study. J Am Coll Cardiol. 2001;38:105-10.

44. Hoffmann U, Bamberg F, Chae CU, et al. Coronary computed tomography angiography for early triage of patients with acute chest pain: the ROMICAT (Rule Out Myocardial Infarction using Computer Assisted Tomography) trial. J Am Coll Cardiol. 2009;53:1642-50.

45. Nabi F, Chang SM, Pratt CM, et al. Coronary artery calcium scoring in the emergency department: identifying which patients with chest pain can be safely discharged home. Ann Emerg Med. 2010;56:220-9.

46. Becker A, Knez A, Becker C, et al. Prediction of serious cardiovascular events by determining coronary artery calcification measured by multi-slice computed tomography]. Dtsch Med Wochenschr. 2005;130:2433-8.

47. Detrano R, Hsiai T, Wang S, et al. Prognostic value of coronary calcification and angiographic stenoses in patients undergoing coronary angiography. J Am Coll Cardiol. 1996;27:285-90.

48. Keelan PC, Bielak LF, Ashai K, et al. Long-term prognostic value of coronary calcification detected by electron-beam computed tomography in patients undergoing coronary angiography. Circulation. 2001;104:412-7.

49. Laudon DA. Computed tomographic coronary artery calcium assessment for evaluating chest pain in the emergency department: longterm outcome of a prospective blind study. Mayo Clin Proc. 2010.

50. McLaughlin VV, Balogh T, Rich S. Utility of electron beam computed tomography to stratify patients presenting to the emergency room with chest pain. Am J Cardiol. 1999;84:327-8. A8.

51. Rozanski A, Gransar H, Wong ND, et al. Clinical outcomes after both coronary calcium scanning and exercise myocardial perfusion scintigraphy. J Am Coll Cardiol. 2007;49:1352-61.

52. Schenker MP, Dorbala S, Hong EC, et al. Interrelation of coronary calcification, myocardial ischemia, and outcomes in patients with intermediate likelihood of coronary artery disease: a combined positron emission tomography/computed tomography study. Circulation. 2008;117:1693-700.

53. Schmermund A, Stang A, Mohlenkamp S, et al. Prognostic value of electron-beam computed tomography-derived coronary calcium scores compared with clinical parameters in patients evaluated for coronary artery disease. Prognostic value of EBCT in symptomatic patients. Z Kardiol. 2004;93:696-705.

54. Kwon SW, Kim YJ, Shim J, et al. Coronary artery calcium scoring does not add prognostic value to standard 64-section CT angiography protocol in low-risk patients suspected of having coronary artery disease. Radiology. 2011;259:92-9.

55. Cho I, Chang HJ, Sung JM, et al. Coronary computed tomographic angiography and risk of all-cause mortality and nonfatal myocardial infarction in subjects without chest pain syndrome from the CONFIRM Registry (coronary CT angiography evaluation for clinical outcomes: an international multicenter registry). Circulation. 2012;126:304-13.

56. Taylor AJ, Cerqueira M, Hodgson JM, et al. ACCF/SCCT/ACR/ AHA/ASE/ASNC/NASCI/SCAI/SCMR 2010 Appropriate Use Criteria for Cardiac Computed Tomography. A Report of the American College of Cardiology Foundation Appropriate Use Criteria Task Force, the Society of Cardiovascular Computed Tomography, the American College of Radiology, the American Heart Association, the American Society of Echocardiography, the American Society of Nuclear Cardiology, the North American Society for Cardiovascular Imaging, the Society for Cardiovascular Angiography and Interventions, and the Society for Cardiovascular Magnetic Resonance. J Cardiovasc Comput Tomogr. 2010;4:407-33.

57. Villines TC, Carbonaro S, Hulten E. Calcium scoring and chest pain: is it dead on arrival? J Cardiovasc Comput Tomogr. 2011;5:30-4.

58. Strong JP, Malcom GT, McMahan CA, et al. Prevalence and extent of atherosclerosis in adolescents and young adults: implications for prevention from the Pathobiological Determinants of Atherosclerosis in Youth Study. JAMA. 1999;281:727-35.

59. Cheezum MK, Hulten EA, Taylor AJ, et al. Cardiac CT angiography compared with myocardial perfusion stress testing on downstream resource utilization. J Cardiovasc Comput Tomogr. 2011;5:101-9.

60. Garber AM, Solomon NA. Cost-effectiveness of alternative test strategies for the diagnosis of coronary artery disease. Ann Intern Med. 1999;130:719-28.

61. Halpern EJ, Fischman D, Savage MP, Koka AR, DeCaro M, Levin DC. Decision analytic model for evaluation of suspected coronary disease with stress testing and coronary CT angiography. Acad Radiol. 2010;17:577-86.

62. Kuntz KM, Fleischmann KE, Hunink MG, Douglas PS. Costeffectiveness of diagnostic strategies for patients with chest pain. Ann Intern Med. 1999;130:709-18.

63. Lorenzoni R, Cortigiani L, Magnani M, et al. Cost-effectiveness analysis of noninvasive strategies to evaluate patients with chest pain. J Am Soc Echocardiogr. 2003;16:1287-91.

64. Marwick TH, Shaw L, Case C, Vasey C, Thomas JD. Clinical and economic impact of exercise electrocardiography and exercise echocardiography in clinical practice. Eur Heart J. 2003;24:1153-63.

65. Min JK, Kang N, Shaw LJ, et al. Costs and clinical outcomes after coronary multidetector CT angiography in patients without known coronary artery disease: comparison to myocardial perfusion SPECT. Radiology. 2008;249:62-70.

66. Min JK, Gilmore A, Budoff MJ, Berman DS, O'Day K. Costeffectiveness of coronary CT angiography versus myocardial perfusion SPECT for evaluation of patients with chest pain and no known coronary artery disease. Radiology. 2010;254:801-8.

67. Otero HJ, Rybicki FJ, Greenberg D, Mitsouras D, Mendoza JA, Neumann PJ. Cost-effective diagnostic cardiovascular imaging: when does it provide good value for the money? Int J Cardiovasc Imaging. 2010;26:605-12. 